\title{
RELATO DE EXPERIÊNCIA SOBRE O PROCESSO EDUCATIVO PARA A PROMOÇÃO DA SAÚDE DE ADOLESCENTES
}

\section{EXPERIENCE'S REPORT ABOUT EDUCATIONAL PROCESS OF ADOLESCENT'S HEALTH PROMOTION}

\author{
Luiza Akiko Komura Hoga* \\ Catarina Terumi Abe**
}

HOGA, L.A.K.; ABE, C.T. Relato de experiência sobre o processo educativo para a promoção da saúde de adolescentes. Rev.Esc.Enf.USP, v. 34, n.4, p. 407-12, dez. 2000.

\section{RESUMO}

Este artigo trata-se de um relato de experiencia sobre um trabalho desenvolvido com um um grupo de adolescentes, moradores em uma comunidade de baixa renda. O objetivo do trabalho foi a educação para a saúde com contextualização socioeconômica e cultural. Participaram do grupo, 34 adolescentes de ambos os sexos, com idades entre 12 e 18 anos. Foram realizadas nove sessões grupais de educação para a saúde, no periodo entre setembro e dezembro de 1997. Foram levantadas as principais dúvidas que os adolescentes tinham sobre saúde, que foram esclarecidas no decorrer das sessões de educação para a saúde, com utilização de estratégia participativa, associados a vários recursos didáticos. Os termos técnicos foram decodificados para a linguagem popular. Com base nos temas trabalhados nas sessões, foi elaborado um caderno educativo e a compreensão sobre o conteúdo foi validado com os adolescentes. A experiência permitiu conhecer as peculiaridades do grupo e planejar orientações compreensiveis e significativas aos adolescentes

PALAVRAS-CHAVE: Promoção da Saúde. Adolescência. Grupos.

\begin{abstract}
This article is a experience report about a group developed with adolescents, living in a low income community in sao Paulo City. The aim of the task was the adolescent's health promotion, with sociocultural contextualization for this group. Took part in 34 adolescents of both sex, from 12 to 18 years old. It was realized nine health classes when the adolescents reported their doubts related to health. The methodology of the work was based on participative approach during the educational process. The adolescent's doubts about health were classified according to the similarities, and answered according to the scientific literature. These answers were formulated in popular language. The educational resource's contents were validated with the adolescents and they have suggested the necessary modifications. The experience permitted to know the charactheristics of this particular group and to plan a kind of orientations meaningful to the adolescents.
\end{abstract}

KEYWORDS: Health promotion. Adolescence. Groups.

\section{INTRODUÇÃO}

As questões inerentes a uma fase muito importante da vida do ser humano - o adolescer têm sido o foco de atenção de muitos profissionais, e também dos próprios pais. Atualmente, professores e profissionais da área da saúde e das ciências sociais, num esforço conjunto, desenvolvem projetos com a finalidade de proporcionar aos adolescentes, uma transição saudável da infância à idade adulta.

Os enfermeiros, por serem agentes de promoção da saúde, também vêm desenvolvendo trabalhos assistenciais e de pesquisa na área, visto as inúmeras publicações científicas enfocando a temática da

\footnotetext{
* Enfermeira Obstétrica. Professora Doutora do Departamento de Enfermagem Materno-Infantil e Psiquiátrica da Escola de Enfermagem da Universidade de São Paulo-EEUSP. Email: lakhoga@sti.com.br

** Enfermeirá' Obstétrica. Ex-Bolsista de Iniciação Científica (Pibic-CNPq)
} 
adolescência (CALDAS,1991; CADETE, 1994; OLIVEIRA, 1997). Estes pesquisadores consideram a necessidade de realizar um exame reflexivo das ações de assistência à saúde desenvolvidas com os adolescentes, para compreender o universo de saúde deles e proporcionar-lhes respostas empáticas, compreensivas e seguras a seus problemas e questionamentos.

ROGA (1988) dedicou-se ao estudo de temas básicos da educação à saúde do jovem, tais como crescimento, desenvolvimento e agravos à saúde. Constatou que os jovens tinham tido pouca ou quase nenhuma oportunidade de discutir e compartilhar seus anseios em relação aos conhecimentos sobre as transformações ocorridas durante o adolescer. Descreveu também o cotidiano de mulheres moradoras em uma comunidade de baixa renda e verificou que elas não possuem uma fonte segura e confiável para sanar suas dúvidas relativas a seus corpos, sobretudo sobre as transformações pelas quais passaram durante a fase da adolescência. As mulheres relataram que se sentiram "atropeladas" por todas as transformações que ocorreram rapidamente em seus corpos e pelas vivências próprias daquela fase da vida, o que se deveu ao despreparo em que se encontravam, pois não tinham com quem falar a respeito (ROGA, 1995). Existe, portanto, um vácuo na promoção de saúde do adolescente que merece maior atenção de todos os envolvidos nesta questão.

Diversas publicações, sejam elas do meio acadêmico ou não, permitem constatar que os adolescentes convivem com uma série de questionamentos sobre as transformações que estão ocorrendo consigo, e necessitam ser abordados, tanto individualmente como no âmbito coletivo.

No decorrer das práticas educativas, pode ser sentida a complexidade de tudo aquilo que envolve a decodificação da linguagem científica para a popular, dos conteúdos inerentes ao conhecimento do corpo humano e das práticas de saúde e da forte influência exercida pelo meio social e cultural. Estes fatores, intrínsicamente relacionados, devem ser devidamente conhecidos, considerados e trabalhados, pois eles são essenciais à comunicação e troca eficiente de idéias entre os interlocutores envolvidos em um processo educativo em saúde.

Avalia-se que o desenvolvimento destas atividades é facilitado e beneficiado se lhes for oferecido um material educativo, com linguagem e recursos audiovisuais com os quais os adolescentes possam identificar-se. Acredita-se que a existência de material educativo contendo orientações e esclarecimentos sobre a saúde, com tais características possa trazer grandes beneficios tanto aos adolescentes como aos profissionais que se propõem realizar este tipo de atividade.
O Estatuto da Criança e do Adolescente (SÃO PAULO, 1993) considera o desenvolvimento sadio e harmonioso da criança e do adolescente como um dos direitos fundamentais para esta faixa etária, e sugere oferecimento de orientação destinados especificamente a eles.

Os membros assessores da Organização Mundial da Saúde (WHO, 1996), corroboram esta idéia, especialmente, para países em desenvolvimento. Observam que, para promoção da saúde dos adolescentes, seja importante envolvê-los desde o planejamento dos programas até sua avaliação. Salientam que o enfoque mais adequado é a contextualização do processo educativo ao meio sociocultural onde os adolescentes vivem.

Acreditando que os anseios dos jovens devem ser conhecidos e trabalhados de forma adequada e contextualizada na perspectiva social, cultural e segundo a capacidade cognitiva de cada pessoa, as autoras desenvolveram sessões grupais com adolescentes, que teve como linha norteadora a abordagem participativa visando a educação e a promoção da saúde. Os grupos foram conduzidos com ênfase na preservação da identidade sociocultural dos próprios adolescentes, e no cuidado voltado à utilização de terminologia e material audiovisual avaliado como o mais adequado à compreensão do conteúdo trabalhado em cada sessão grupal:

O objetivo deste artigo é relatar a experiência das autoras na condução de um grupo de adolescentes, desenvolvido com metodologia participativa, na qual procurou-se preservar a identidade social e cultural dos componentes do grupo. Ao final das sessões grupais foi elaborado um material educativo voltado à promoção da saúde dos adolescentes. $\mathrm{O}$ conteúdo deste material foi validado pelos próprios adolescentes, quanto à pertinência e facilidade de compreensão do mesmo.

\section{O LOCAL E A POPULAÇÃO PARTICIPANTE DO GRUPO}

As atividades de educação para a saúde foram realizadas com adolescentes moradores de uma comunidade de baixa renda, localizada na Zona Oeste da Região Metropolitana de São Paulo, onde os membros do Núcleo de Assistência para o Autocuidado da Mulher (NAAM), realizam atividades de pesquisa e promoção dos diversos aspectos da saúde.

As sessões ocorreram no período de setembro a dezembro de 1997, nas dependências de uma Congregação Religiosa, situada nas proximidades da comunidade que mantém um projeto denominado Centro da Juventude (CJ), em parceria com o Governo Municipal. Este tem por objetivo manter os 
jovens ocupados com atividades esportivas, reforço escolar, entretenimento, convivência social, entre outras. O grupo foi composto por 34 adolescentes, na faixa etária entre 12 e 18 anos, sendo 13 do sexo feminino e 21 do masculino.

\section{AS ETAPAS DESENVOLVIDAS NO GRUPO}

A proposta inicial do trabalho estabelecia que ele seria desenvolvido na própria comunidade. Nos contatos iniciais com os adolescentes em seus domicílios, pôde-se perceber que eles encontravamse diariamente no $\mathrm{CJ}$ referido, o que levou as pesquisadoras a realizarem o trabalho naquele local.

Estabeleceu-se contato preliminar com o responsável pelo CJ. Este aprovou prontamente a nossa idéia, pois também avaliava que os adolescentes necessitavam conhecer e cuidar melhor da saúde deles. Foi esclarecido ao responsável que, concomitantemente, às sessões de educação para a saúde, seria também elaborado um material educativo, a ser construído no decorrer do desenvolvimento dos trabalhos grupais. Foi esclarecido que necessitar-se-ia da participação contínua dos adolescentes para exposição de seus questionamentos relativos à saúde.

As perguntas que surgissem, seriam respondidas e levadas ao conhecimento dos próprios jovens para que estes validassem o material produzido, quanto à clareza e facilidade de compreensão, assim como quanto à pertinência das ilustrações. A idéia foi levada aos adolescentes, que também se empolgaram com a proposição do estudo. Proporcionou-se liberdade para participar ou não das sessões-trabalho e alguns preferiram desenvolver outras atividades próprias do CJ.

Obedeceu-se aos itens constantes na Resolução 196-96 que trata das Normas de Pesquisa Envolvendo Seres Humanos (BRASIL, 1996). Obtevese consentimento livre e esclarecido dos adolescentes participantes com um esclarecimento preliminar dos objetivos e finalidades da pesquisa, a forma como seria desenvolvida, a liberdade de participação ou não, e a garantia de sigilo e anonimato.

$\mathrm{Na}$ primeira sessão com os jovens, ocorrida no final de agosto de 1997, as duas pesquisadoras, autoras deste artigo, apresentaram os objetivos gerais e específicos do estudo. Foi-lhes oferecida a oportunidade para expor as dúvidas que eles tinham em relação a algum aspecto da saúde, da maneira que julgassem mais conveniente. Os adolescentes optaram por expor suas dúvidas oralmente. Eles também desejaram dividir o grupo segundo o sexo, sugestão esta respeitada pelas educadoras. Assim sendo, primeiramente se realizava a sessão grupal com um determinado sexo e, na seqüência, com o outro. Em todas as sessões subsequentes foi adotado o mesmo critério, e contou com a presença das duas pesquisadoras.

Adotou-se uma pergunta descritiva ampla: "Falem o que vocês querem saber sobre sua saúde". Apresentada a questão, observou-se dificuldade na exposição das dúvidas e sentiu-se necessidade de mudança da estratégia. Foi sugerido que na sessão seguinte seria oferecida uma orientação preliminar de conteúdos básicos relativos a uma temática eleita previamente pelo grupo. Aceita a idéia, assim se procedeu nas sessões subseqüentes.

A estratégia permitiu o contato direto do adolescente com alguns conteúdos específicos de saúde, muitos deles até então desconhecidos. Gerou oportunidade para refletir o assunto trabalhado, tendo facilitado o desencadeamento de novos questionamentos.

Simultaneamente aos diálogos explicativos com os adolescentes, surgiram novas dúvidas, que iam sendo esclarecidas por uma das pesquisadoras, enquanto a outra anotava todos os novos questionamentos aparecidos no transcorrer dos trabalhos. A estratégia facilitava a exposição de dúvidas consideradas pelos próprios adolescentes como de dificil expressão.

Os recursos didáticos adotados em cada sessão foram selecionados segundo o critério da adequação ao conteúdo a ser trabalhado naquele dia. Foram utilizadas a exposição dialogada, várias dinâmicas grupais e filmes comentados. Realizaram-se nove sessões, com presença média de dez adolescentes em cada uma. Pelo fato dos jovens terem optado pela divisão do grupo segundo o sexo, ocorriam duas sessões no mesmo dia, uma subseqüente a outra. O tempo médio de duração de cada uma foi 1 h30.

Após o encerramento das atividades realizadas em grupo com os adolescentes, as dúvidas trabalhadas em todas as sessões, tanto com o grupo feminino como o masculino, foram organizadas para compor um material educativo. Este trabalho foi realizado de acordo com as seguintes etapas:

- Preparação de respostas às perguntas dos adolescentes, com base na literatura científica; -

Classificação e agrupamento das mesmas, segundo o critério da similaridade;

- Decodificação de linguagem, na ótica das próprias pesquisadoras, da científica à popular com adoção de termos utilizados pelos próprios adolescentes; Elaboração de ilustrações relativas aos conteúdos, para facilitar a compreensão das respostas, aos olhos dos adolescentes;

- Avaliação do material pelos adolescentes para sugestão das alterações pertinentes;

- Revisão do material, conforme sugerido e levado novamente a apreciação, procedimento este repetido até a validação final do material pelos adolescentes. 
Os principais tópicos que foram alvo dos interesses dos adolescentes de ambos os sexos foram sobre o conhecimento do corpo e do funcionamento do mesmo; a capacidade reprodutiva do ser humano e o controle da fertilidade; o aborto e as conseqüências de sua realização, sob o ponto de vista físico, legal e de saúde mental; o uso da camisinha, as doenças que pegam por meio do sexo e AIDS; as conseqüências do uso do álcool e das drogas; e sobre a ocorrência e a evolução de uma gravidez. Todos estes tópicos foram discutidos no trancorrer do desenvolvimento dos grupos, inseridos dentro de uma conjuntura socioeconômica e cultural, muito embora o material produzido contivesse apenas as "respostas" pontuais às questões formuladas pelos adolescentes.

\section{CONSIDERAÇÕES FINAIS}

O desenvolvimento deste trabalho com grupo de adolescentes permitiu constatar que para a formação e condução de grupos de educação para a saúde há a necessidade de uma coordenação, realizada de forma sistemática e de instalações fisicas apropriadas.

Ao introduzir uma proposta de trabalho com adolescentes, é imprescindivel que seja claramente observado o objetivo da mesma, assim como a importância do envolvimento de todas as pessoas participantes do processo. O desligamento, mesmo que parcial, de alguns dos integrantes do grupo reflete-se no processo e no resultado final do trabalho. A fase de validação, pelos jovens, do material educativo produzido teve dificuldades em razão dos responsáveis pelo $\mathrm{CJ}$ terem adotado o critério de 14 anos como a idade limite de permanência no projeto. Quando se retornou ao local para realizar a etapa de validação do texto e das ilustrações, os adolescentes maiores de 14 anos já haviam sido desligados. Foi necessário procurá-los individualmente em seus domicilios. Constatou-se que o contato individual foi um fator restritivo, pois os jovens mostravam-se mais entusiasmados, abertos ao diálogo e à troca em ambiente coletivo, onde pairavam a coesão grupal e a possibilidade de identificarem-se mutuamente. Uma vez desfeito o grupo de adolescentes diluíram-se também os beneficios proporcionados pela identificação como membro de um grupo de adolescentes.

Quando os educandos encontram-se em estado de total falta de conhecimento do assunto, qualquer material por mais acessivel que seja no aspecto da facilidade de compreensão, não atinge seu objetivo pelo fato de estar descontextualizado. Isto foi constatado na condução deste trabalho. Quando se sugeriu a oportunidade aos adolescentes de manifestarem suas dúvidas sobre a saúde, as pesquisadoras foram surpreendidas pela dificuldade demonstrada por eles ao formulá-las. A situação foi justificada pelos próprios jovens, pois não tiveram acesso aos conhecimentos relativos à saúde e, conseqüentemente, não poderiam ter dúvidas de algo que desconheciam.

O reconhecimento desta realidade levou à proposição de vários temas que foram sugeridos aos adolescentes e, após, aceitos por eles foram abordados nas sessões subseqüentes. As aulas expositivas com temática eleita pelos próprios jovens foram fundamentais à formulação das dúvidas. Possibilitaram constatar, ainda, que o material produzido deve estar associado a um processo educativo em que é importante a presença de um educador como facilitador da aprendizagem. Portanto, o material educativo constitui-se em recurso adicional, mas não substitui o educador.

Outro aspecto diz respeito à baixa escolaridade formal dos adolescentes. Este déficit educacional restringe a capacidade de compreensão de muitos termos comumente utilizados na área da saúde e requer do educador, um trabalho minucioso de decodificação da linguagem. Os profissionais que desenvolvem atividades de educação para a saúde precisam estar atentos a sua comunicação porque muitos termos comumente empregados no cotidiano, considerados de fácil entendimento na avaliação das pesquisadoras, podem estar sendo muito dificeis sob o ponto de vista das pessoas pouco acostumadas à leitura e à reflexão. Há que se considerar também que a educação para a saúde deve vincular-se a um processo amplo de formação de atitudes, pois se oferecida de forma isolada, pode provocar no adolescente sentimentos de insegurança, impotência e ansiedade (GOJMAN, 1995).

Ao visualizar o processo educativo e a elaboração do material educativo dentro de um contexto maior torna-se evidente que alguns assuntos relativos à saúde, chamaram a atenção dos adolescentes, visto o interesse demonstrado por eles. Estes, porém, não se constituem em preocupação nem necessidade primordial destes jovens. Ao conduzir a presente pesquisa, tornou-se evidente que a grande problemática enfrentada por estes adolescentes situa-se na esfera socioeconômica, em especial, na falta de expectativas positivas em relação ao próprio futuro. A ausência de um rumo definido, ou pelo menos levemente delineado para suas vidas, colocaos frente a uma indefinição na trajetória a seguir, afetando-os em muitos aspectos, sobretudo no tocante à saúde mental.

Um estudo etnográfico conduzido por HOGA, ABE (1997) nesta mesma comunidade permitiu constatar que estes adolescentes levam um tipo de vida ao qual devem estar constantemente atentos 
para o fato de "Ter de possuir muito cuidado para a mente não desandar", pois muitos e constantes são os estímulos para que estes adolescentes "desandem" e prossigam na prática de contravenções e, conseqüente, vida na marginalidade.

Is to reforça a idéia preconizada por FRIEDMAN (1993) de que o processo saúde-doença insere-se numa dimensão muito ampla, no qual todos os fatores presentes encontram-se em interação. Recomenda não visualizar os jovens como sujeitos portadores de "problemas" em potencial, porque este enfoque é restritivo e leva a soluções específicas e limitadas a questões pontuais. FERRAROTI (1985) também concorda que as ações educativas não devem ser limitadas a áreas demarcadas, pois a saúde plena abrange inúmeros aspectos. Ressalta que, especificamente na fase da adolescência, há determinadas áreas em que a educação para a saúde é imprescindivel como, por exemplo, a sexual, alimentar e higiênica, pois é na adolescência que surgem as curiosidades a respeito destes assuntos.

Mesmo corroborando a idéia destes especialistas sobre a amplitude em que se insere o processo saúde-doença e a necessidade de levar-se em consideração todos os fatores envolvidos, registramos aqui o parecer acerca a importância das intervenções voltadas à promoção da saúde, durante a adolescência. Concorda-se com KRAUSKOPF (1995) para quem tal espaço permite a expressão de idéias, dúvidas, sentimentos sobre crescimento e imagem corporal e consciência da própria inserção social, e também quando este autor refere-se à idade dos componentes do grupo, que não precisam ser necessariamente homogêneos. A vivência das pesquisadoras permite afirmar que as trocas de experiências entre adolescentes de diferentes idades é bastante salutar, em certos aspectos, como ocorreu neste processo educativo.

A participação de ambos os sexos no grupo possibilitando a interação entre eles, é sugerida por KRAUSKOPF (1995), que embora visualize isto ser variável de acordo com a cultura, constitui-se em momento benéfico ao estímulo, à participação do sexo masculino, que na experiência analisada, tende a participar menos nas atividades grupais.

Neste grupo de adolescentes, foi ofertada a oportunidade de participação de ambos os sexos num mesmo grupo e foram ressaltados os beneficios inerentes a esta prática. Todavia tal alternativa foi frontalmente combatida, notadamente, por parte das jovens que se recusaram a integrar um grupo com participação masculina. Elas alegavam que se sentiam inibidos com a presença de pessoas do sexo oposto e, e nestas cinscunstâncias, não sentiam-se livres para fazer perguntas de foro intimo e relacionadas ao sexo feminino.
Como pesquisadoras-observadoras do contexto, considerou-se que esta forma de conduta possui raizes mais profundas, visto que nesta comunidade a relação entre os gêneros é bastante conflituosa. Isto pôde ser percebido no decorrer das sessões de educação para a saúde, quando as jovens expuseram muitos exemplos de subordinação à dominação masculina.

A igualdade de gêneros ainda se encontra em fase desafiadora, na maior parte do mundo (MUNDIGO, 1995). Na visão deste autor, ainda nos encontramos em fase de compreensão de sua gênese, pois não se tem clareza das ocorrências intrafamiliares no transcorrer das gerações, na transmissão de normas sexuais, nas posições de poder e na tipificação do comportamento dos gêneros.

No que se refere ao gênero, observou-se que na perspectiva feminina, existe uma clara noção entre as jovens sobre a necessidade que elas têm de adequar os seus comportamentos e atitudes aos valores de gênero existentes na comunidade, predominantemente centrados na perspectiva masculina. Mostram-se, em geral, passivas e conformadas com o etnocentrismo masculino nas questões de gênero.

Segundo o que foi salientado até então, visualizase que há muito por fazer para a saúde do adolescente. Os especialistas da Organização Mundial da Saúde (WHO, 1996) identificaram princípios vitais de uma ação efetiva e a importância do envolvimento do jovem no planejamento, implementação e avaliação das ações, considerando os beneficios, envolvimento multisetorial e multidisciplinar e o enfoque personalizado, segundo o contexto social.

A concretização destes princípios requer, pelo lado do profissional de saúde, dentre eles o enfermeiro(a), a transposição dos obstáculos impeditivos para a boa saúde do adolescente, que segundo FRIEDMAN (1993) necessitam de conhecimentos adequados das fases de amadurecimento, especialmente, no que refere à sexualidade; capacitação dos próprios profissionais em temas relacionados à sexualidade e comunicação com os adolescentes: inclusão dos jovens no planejamento e avaliação dos serviços; enfoque claro e construtivo das necessidades dos adolescentes, na politica e legislação relativa a eles.

A guisa de conclusão, reitera-se que a presente iniciativa, de elaborar um material educativo para promoção de saúde do adolescente contextualizado socioculturalmente foi uma experiência construtiva, gratificante e viável, muito embora considere-se que muito há a ser conhecido e realizado na área. O conhecimento adquirido nesta experiência impulsiona as pesquisadoras a prosseguirem neste caminho e a enfrentarem novos desafios, em prol da saúde dos adultos do futuro. 


\section{REFERÊNCIAS BIBLIOGRÁFICAS}

BRASIL. MINISTÉRIO DA SAÚDE. CONSELHO Nacional de Saúde. Resolução n.196 de 10 de outubro de 1996: Diretrizes e normas regulamentadoras de pesquisas em seres humanos. Mundo Saúde, v.21, n.1, p.52-61, 1996.

CADETE, M.M.M. Da adolescência ao processo de adolescer. São Paulo, 1994. 140p. Tese (Doutorado) - Escola de Enfermagem, Universidade de São Paulo.

CALDAS, M.A. de M. Pensamentos e experiências na área de saúde de pessoas que vivenciam o adolescer: Uma abordagem fenomenológica. São Paulo, 1991. 186p. Dissertação (Mestrado) - Escola de Enfermagem, Universidade de São Paulo.

FERRAROTTI, N.G. La salud y Ias áreas de'educación para Ia salud en Ia adolescência. In: MADDALENO, et al. La salud del adolescent $y$ del joven. Washington, Organización Panamericana de Ia Salud. 1985. p. 30-6. (Publicación científica $n$. 489)

FRIEDMAN, H.L. Promoting the health of adolescents in the United States of America: a global perspective. J. Adolesc.Health. v. 14, n.7, p.509-19,1993.

gojman, I. Promoción de salud en Ia escuela. In: MADDALENO, et al. La salud del adolescent y del joven. Washington, Organización Panamericana de Ia Salud. 1995. p. 46-56 (Publicación científica n. 552).

HOGA, L.A.K. A prevenção da gravidez na adolescência proposta por estudantes de segundo grau. São Paulo, 1988 117p. Dissertação (Mestrado) - Escola de Enfermagem, Universidade de São Paulo.

. À mercê do cotidiano da anticoncepção: a mulher seguindo o seu caminho. São Paulo, 1995. 247p. Tese (Doutorado) Escola de Enfermagem, Universidade de São Paulo.
HOGA, L.A.K.; ABE, C.T. Ser adolescente em uma comunidade de baixa renda da Cidade de São Paulo: um estudo etnográfico./ Apresentado ao 49. Congresso Brasileiro de Enfermagem, Belo Horizonte, 1997/.

KRAUSKOPF, D. Los grupos de pares en Ia adolescencía. In: MADDALENO, et al. La salud deL adolescent y del joven. Washington, Organización Panamericana de La Salud. 1995. p.118-24. (Publicación científica n. 552)

MUNDIGO, A.I. Papéis masculinos, saúde reprodutiva e sexualidade. São Paulo, Fundação Mac Arthur, 1995.

OLIVEIRA, M.A. de C. A adolescência, o adolescer e o adolescente: re-significação a partir da determinação social do processo saúde-doença. São Paulo, 1997. 142p. Tese (Doutorado) - Escola de Enfermagem, Universidade de São Paulo.

SÃO PAULO (Estado). Fundo Social de Solidariedade. Direitos da criança e do adolescente. São Paulo, 1993. 105p.

WORLD HEALTH ORGANIZATION. Family \& reprodutive health. Genebra, 1996. 